\title{
Structure analysis of immobilized-bovine serum albumin by means of TOF-SIMS
}

\author{
Satoka Aoyagi* and Makoto Dohi \\ Faculty of Life and Environmental Science, Shimane University, \\ 1060 Nishikawatsu-cho, Matsue-shi, Shimane, 690-8504, Japan \\ Nobuhiko Kato and Masahiro Kudo \\ Department of Materials and Life science, Seikei University, Japan \\ Shinichi Iida, Miyako Tozu, and Noriaki Sanada \\ ULVAC-PHI, Inc., Japan
}

(Received 26 July 2006; Accepted 17 August 2006; Published 29 August 2006)

\begin{abstract}
Orientation of immobilized proteins on bio-devices is important to obtain their high performance. Structural change in a particular area of a protein is also very important for the study of the protein performance and the evaluation of the bio-devices. Time-of-flight secondary ion mass spectrometry (TOF-SIMS) is able to analyze upper surface of one layer of molecules. Orientation of immobilized proteins can be evaluated based on determination of a partial structure, representing ensemble of amino acids, on the surface part. The model protein, bovine serum albumin (BSA), is immobilized on the substrate, indium-tin oxide (ITO) glass electrode, by covalent bonding. Two types of the oriented samples were prepared by controlling the binding parts of BSA. The results from TOF-SIMS spectra analysis were compared to the amino acid sequence to examine surface parts of the immobilized BSA at different groups. The surface parts of the BSA molecules immobilized on ITO glass plates with different parts of the molecule, amino groups or carboxyl groups, are evaluated by means of TOF-SIMS. The orientation differences were clearly shown in TOF-SIMS spectra of the samples. Furthermore, fragment-ion-generating parts of immobilized-BSA are determined by fragment ions of particular combinations of amino acids in the sequence of BSA. [DOI: 10.1380/ejssnt.2006.614]
\end{abstract}

Keywords: TOF-SIMS; structure analysis; surface analysis; albumin

\section{INTRODUCTION}

Orientation of immobilized proteins on bio-devices $[1,2]$ is important to obtain their high performance. Structural change in a particular area of a protein is also very important for the study of the protein performance and the evaluation of the bio-devices. Analysis of protein structure has been studied in many fields such as x-ray diffraction analysis, NMR, Raman and Infrared spectroscopy $[3-5]$ and mass spectrometry $[6,7]$. Though the spectroscopy determines chemical structures of molecules, it is difficult to analyze partial structure because proteins have the same groups. In addition, these techniques require more than one layer of molecules on substrates. An analysis method with high sensitivity and high resolution is required in order to evaluate a bio-device with bio-molecules.

Time-of-flight secondary ion mass spectrometry (TOFSIMS) is one of the most sensitive surface analysis techniques [8]. TOF-SIMS is able to provide information on orientation and partial structure of immobilized protein based on fragment ions composed of multiple amino acids, in principle. TOF-SIMS analyzes upper surface of one layer of molecules. Orientation of immobilized proteins can be evaluated based on determination of a partial structure, indicating ensemble of amino acids, on the surface part. Large fragment ions consisting of multiple amino acids will indicate which part of a protein is on the surface. There are probably specific and important fragment ions to determine the surface part of a molecule,

*Corresponding author: aoyagi@life.shimane-u.ac.jp because current TOF-SIMS techniques produce a variety of fragment ions. Since ion generation mechanism of SIMS has not been clarified yet, fragment ions from complicated samples such as proteins cannot be predicted. Therefore analysis techniques for TOF-SIMS spectra interpretation will be helpful to find out important peaks. In this study, spectra analysis by means of mutual information [9] is employed.

Information theory [10] has been employed to analyze TOF-SIMS data on protein-containing biomaterials [11]. This innovation is now sufficiently developed so as to be able to obtain chemical images of protein samples. Mutual information [10, 12], a technical term defined by information theory, characterizes the specificity of every peak in TOF-SIMS spectra of a sample compared with another sample, such as a reference sample. With mutual information specific, the desired peaks can be selected out of a great number of peaks which appear in TOF-SIMS spectra.

TOF-SIMS has been applied to biomaterial analysis [9, 13-19] to evaluate adsorbed protein films [20], protein folding and orientation $[21,22]$, and protein distribution onto medical devices [10, 23-25] and to study extracellular matrix [26, 27] and plants [28-30]. Protein distribution on artificial kidney and biosensors were evaluated in the previous studies [24, 31]. Peaks of fragment ions specific to a particular protein are found out of all peaks based on values of mutual information calculating from comparison between spectra of a sample and those of reference. Since relatively strong intensity is required to obtain clear images, peaks at higher mass are, sometimes, ignored because of their weak intensities. However, peaks of fragment ions composed of two or three amino acids, which have higher mass values, are important to determine par- 
ticular part of a protein.

In this study, in order to obtain larger fragment ions at high intensities, indium-tin oxide (ITO) glass electrode was employed as a substrate for protein immobilization, because it is often difficult to produce enough fragment ions at higher mass with insulating substrate such as glass. The model protein, bovine serum albumin (BSA), was immobilized on the substrate by covalent bonding. Two types of the oriented samples were prepared by controlling the binding part, i.e., amino groups or carboxyl groups, of the protein.

The primary structure, i.e., the amino acid sequence, of BSA has been reported [32] and its structure is very similar to human serum albumin (HSA) whose three-dimensional structure has already been reported [33] in the Protein data bank (PDB: http://www.rcsb.org/pdb/). The results from TOFSIMS spectra analysis were compared to the amino acid sequence and 3D-strucutre of albumin to examine surface parts of the BSA immobilized in different ways.

\section{MATERIALS AND METHODS}

\section{A. Sample Preparation}

An indium tin oxide (ITO) coated glass plate (SigmaAldrich Co., St Louis, MO, USA) was aminosilanized with aminopropyltrimethoxysilan (Tokyo Kasei, Tokyo, Japan). The aminosilanized glass plate was activated by glutaraldehyde and then soaked in a $0.1 \mathrm{M}$-phosphate buffered saline (PBS) solution at $\mathrm{pH} 7.4$ containing 1 $\mathrm{mg} / \mathrm{ml}$ bovine serum albumin (BSA: Sigma- Aldrich) and allowed to react in the dark for $30 \mathrm{hr}$ at $227 \mathrm{~K}$. After the ITO glass plates were washed in $\mathrm{pH} 7.4$ phosphate buffer saline (PBS), and then they were washed in pure water with sonic waves for $10 \mathrm{~s}$ to remove adsorbed proteins. These ITO glass plates were then rinsed with pure water and dried with freeze dryer (VD-250F, Taitech, Saitama, Japan) before TOF-SIMS measurement.

\section{B. TOF-SIMS}

Positive ion spectra obtained with TOF-SIMS, TRIFTIV (Physical Electronics, Eden Prairie, MN) using $30 \mathrm{kV}$ $\mathrm{Au}_{3}^{+}$primary ion source, were acquired up to $1000 \mathrm{~m} / \mathrm{z}$ while maintaining the primary ion dose at less than $10^{12}$ ions $/ \mathrm{cm}^{2}$ to ensure static conditions. All the spectra, composed of positive ion TOF-SIMS spectra, were calibrated to the $\mathrm{CH}_{3}^{+}, \mathrm{C}_{2} \mathrm{H}_{5}^{+}, \mathrm{C}_{3} \mathrm{H}_{5}^{+}$, and $\mathrm{In}^{+}$peaks before data analysis. Intensities of secondary ion peaks were normalized to their total ion count before calculation of the mutual information in order to correct for the differences in total secondary ion yield from spectrum to spectrum.

\section{Spectrum Analysis}

The calculation steps and the basic concept of classification by means of mutual information were described in the previous paper $[9,24,25]$. A value of information entropy approaches 1 when the peak used for the calculation is important for discriminating samples. On the other hand, a value of information entropy approaches 0 when the peak used for the calculation is not useful for discriminating samples. Calculation steps of the mutual information is the followings:

$$
\begin{aligned}
& S(A)=-\sum p(a i) \log _{2} p(a i), \\
& \text { the probability } p(a i)=\frac{n(a i)}{N}, \quad(i=1,2),
\end{aligned}
$$

where $N$ is the number of all TOF-SIMS spectra, $n(a i)$ is the number of spectra belonging to category $i$, and $S(A)$ is " a priori entropy", information entropy before obtaining information on intensities of a peak.

$$
\begin{aligned}
& S(A \mid B)=-\sum \sum p(b j) p(a i \mid b j) \log _{2} p(a i \mid b j) \\
& \text { the probability } p(a i \mid b j)=\frac{n(a i \mid b j)}{n(b j)} \\
& \qquad j=1 \text { (higher) or } 2 \text { (lower), }
\end{aligned}
$$

where $n(a i \mid b 1)$ is the number of spectra higher than a threshold $V$ out of the spectra belonging to category $i$, $n(b 1)$ is the number of spectra higher than a threshold $V$ out of all spectra, $S(A \mid B)$ is "a posteriori entropy", information entropy after obtaining information on intensities of a peak.

$$
I(A ; B)=S(A)-S(A \mid B),
$$

where $I(A \mid B)$ is mutual information calculated by subtracting a posteriori entropy $S(A \mid B)$ from a priori entropy $S(A)$.

Peaks of secondary ions at $m / z=1$ to 1000 were used for calculation of mutual information.

\section{Peak identification and matching}

Peaks of fragment ions from combinations of amino acids are identified by searching every combination of amino acids based on the following hypothesis: 1) double bonds are not cut, 2) numbers of carbon, oxygen, nitrogen and sulfur are considered, 3) consider SS bonding when there are more than two sulfur atoms. Then the origin of the identified fragment ion, formed combination of amino acids, is found through the primary sequence of BSA.

\section{RESULTS AND DISCUSSION}

Particular fragment ions from combination of multiple amino acids, suggesting orientations of BSA molecules, were searched in the TOF-SIMS spectra of each sample. Table I summarizes the calculation results of the mutual information. There are two types of immobilized BSA: immobilized with amino groups (N-BSA) and immobilized with carboxyl groups (C-BSA). TOF-SIMS spectra of NBSA and those of C-BSA were compared. The peaks at $m / z=259,286,372$ and 413 are specific to N-BSA and the peak at $m / z=129$ is specific to C-BSA as shown in Table I. The peak at $m / z=413$ is not able to be determined because there are too many possibilities. 
TABLE I: Fragment ions specific to each sample. $[\mathrm{AB}] \&[\mathrm{CD}]$ means A \& C, A \& D, B \& C or B \& D.

\begin{tabular}{|c|c|c|c|c|}
\hline Specific to & Peaks & $\begin{array}{l}\text { Mutual infor- } \\
\text { mation }(-)\end{array}$ & Components & Amino acids \\
\hline \multirow[t]{4}{*}{ N-BSA } & 259 & 0.64 & $\mathrm{C}_{15} \mathrm{H}_{35} \mathrm{~N}_{2} \mathrm{O}$ & $\mathrm{W} \&[\mathrm{VK}]$ \\
\hline & 286 & 0.99 & $\mathrm{C}_{17} \mathrm{H}_{38} \mathrm{~N}_{2} \mathrm{O}$ & {$[\mathrm{FY}] \&[\mathrm{FY}]$} \\
\hline & 372 & 0.64 & $\begin{array}{l}\mathrm{C}_{24} \mathrm{H}_{24} \mathrm{~N}_{2} \mathrm{O}_{2} \\
\mathrm{C}_{16} \mathrm{H}_{28} \mathrm{~N}_{4} \mathrm{O}_{4} \mathrm{~S} \\
\mathrm{C}_{19} \mathrm{H}_{24} \mathrm{~N}_{4} \mathrm{O}_{4} \\
\end{array}$ & $\begin{array}{l}\mathrm{M} \& \mathrm{~W} \&[\mathrm{VLIKR}],[\mathrm{FY}] \&[\mathrm{FY}] \& \mathrm{C}, \mathrm{M} \& \mathrm{~K} \& \mathrm{~W} \\
-\end{array}$ \\
\hline & 413 & 0.99 & lots of possibilities & - \\
\hline C-BSA & 129 & 0.99 & $\mathrm{C}_{16} \mathrm{H}_{18} \mathrm{~N}_{2} \mathrm{O}$ & $\begin{array}{l}\mathrm{R}, \mathrm{K}, \mathrm{G} \&[\mathrm{VLIKRP}] \\
{[\mathrm{VLITQKRE}] \&[\mathrm{AVLIMFWSTNQYCKRHDE}]}\end{array}$ \\
\hline
\end{tabular}

The surface part of immobilized BSA is studied comparing the primary sequence of BSA with fragment ions from combination of neighbor amino acids. The sequence for BSA is summarized in the following:

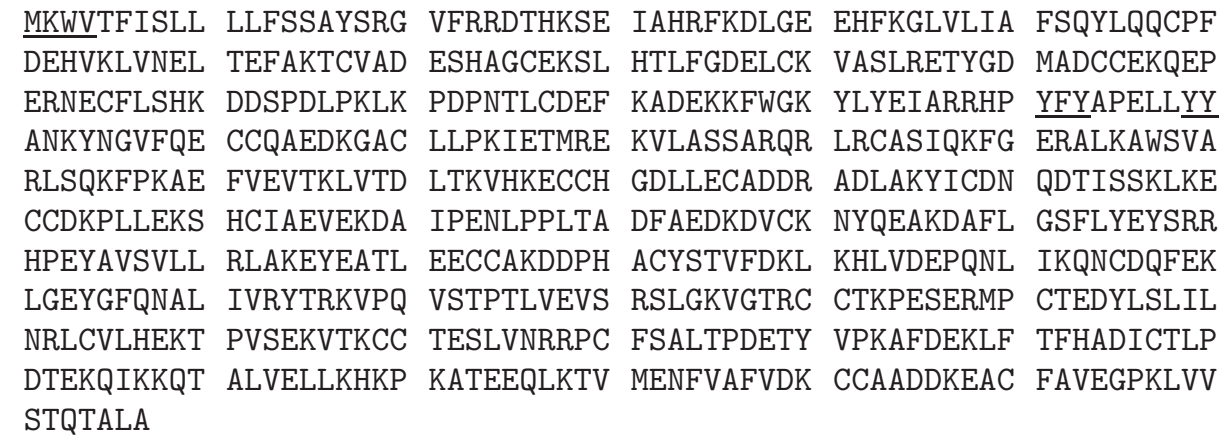

MKWVTFISLL LLFSSAYSRG VFRRDTHKSE IAHRFKDLGE EHFKGLVLIA FSQYLQQCPF

ERNECFLSHK DDSPDLPKLK PDPITLCDEF KADEKKFWGK YLYEIARRHP YFYAPELLYY ANKYNGVFQE CCQAEDKGAC LLPKIETMRE KVLASSARQR LRCASIQKFG ERALKAWSVA RLSQKFPKAE FVEVTKLVTD LTKVHKECCH GDLLECADDR ADLAKYICDN QDTISSKLKE CCDKPLLEKS HCIAEVEKDA IPENLPPLTA DFAEDKDVCK NYQEAKDAFL GSFLYEYSRR HPEYAVSVLL RLAKEYEATL EECCAKDDPH ACYSTVFDKL KHLVDEPQNL IKQNCDQFEK LGEYGFQNAL IVRYTRKVPQ VSTPTLVEVS RSLGKVGTRC CTKPESERMP CTEDYLSLIL STQTALA

Underlined parts, KW (Lysine and Triptophan), WV (Triptophan and Valine), YF(Tyrosine and Phenilalanine), FY, YY, and MKW (Methionine, Lysine and Triptophan) are detected as peaks, at $m / z=259,286$ or 372 , of fragment ions specific to N-BSA. These results indicate that these parts are on the surface or near the surface of N-BSA. Figure 1 shows the 3D structure of human serum albumin (HSA: 1BKE.pdb) having the very similar sequence to BSA. 3D structure of a protein will change depending on its condition. Especially, because these samples have been freeze-dried when they are measured with TOF-SIMS, they will not keep the same structure when they form in crystal. However, this 3D structure suggests, at least, a cue to identify areas facing the surface depending on the binding parts by comparing C-BSA with N-BSA treated in the same processes.

The fragment ions at $m / z=259$ and 286 are produced from the circled parts of the HSA structure shown in Figure 1. Though the KW and WV parts are not shown in Figure 1, these parts will be near the NH2-terminal of the HSA molecule shown in the figure, according to the amino acid sequences of both albumins.

Since the peak at $m / z=129$ specific to C-BSA has lots of possible origins, it is difficult to determine parts which produce the fragment ion. Furthermore, the peak at $m / z=129$ is also observed in the TOF-SIMS spectra of N-BSA due to many possible combinations of amino acids producing the peak in many parts of BSA, though it is mainly specific to C-BSA. Therefore it is difficult to identify the surface part of C-BSA with this peak.

Albumin contains several amino acids having amino groups, i.e., asparagine (N), glutamine (Q), Lysine (K) and arginine (R), or carboxyl groups, i.e., aspartic acid (D) and glutamic acid (E), in the side chains. Therefore, there may be immobilized BSA molecules having different orientations even when they are immobilized at amino groups, because there are so many amino groups in a BSA molecule that it is difficult to identify the parts of BSA binding to the substrates. However, considering the fragment ions related to ensemble of amino acids, at least, it is indicated that most of BSA molecules immobilized with amino groups by covalent bonds have the same orientation placing the square-marked part on the surface of immobilized BSA molecules as shown in Figure 1. In addition, the $\mathrm{NH}_{2}$-terminal of the BSA molecule may not be used in the immobilization processes for N-BSA described in this study, because the $\mathrm{NH}_{2}$-terminal of the BSA molecule may be at the inside of the molecule. Further study is required to obtain detailed information on orientation of immobilized proteins. 


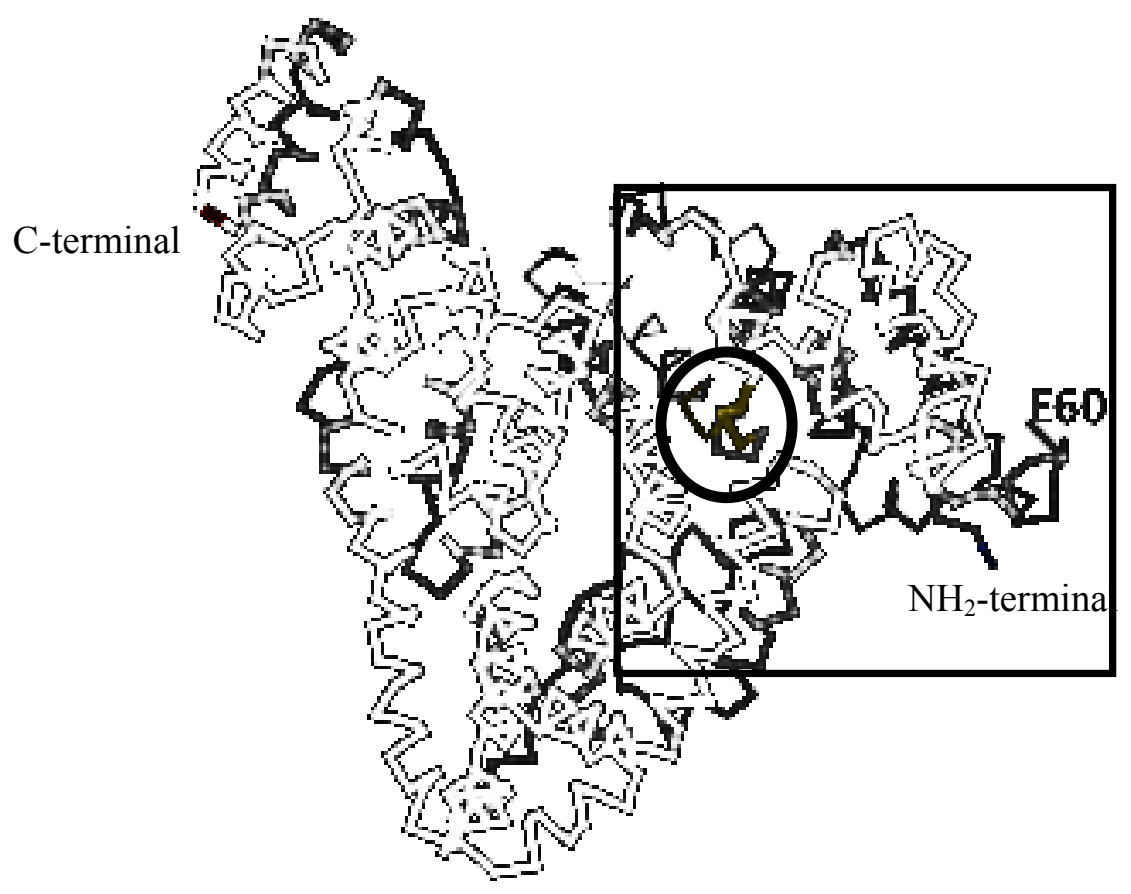

FIG. 1: Structure of human serum albumin. The circled part contains YF, FY, and FF (YY).

\section{CONCLUSIONS}

The surface parts of the BSA molecules immobilized on ITO glass plates with different parts of the molecule, amino groups or carboxyl groups, is evaluated by means of TOF-SIMS. Fragment-ion-generating parts of N-BSA are determined with fragment ions from particular combinations of amino acids in the sequence of BSA, and the surface part of the N-BSA is indicated. Further study is needed to obtain detailed information on orientation of immobilized proteins on bio-devices.
[1] M. Cretich, F. Damin, G. Pirri, M. Chiari, Protein and peptide arrays: Recent trends and new directions, Biomol. Eng. 23, 77-88 (2006).

[2] C. M. Hawlliwell, Nanoanalytical measurement of protein orientation on conductive sensor surfaces, Analyst 129, 1166-1170 (2004).

[3] D. H. Cruz, M.-E. Rousseau, M. M. West, M. Pézolet, A. P. Hitchcock, Quantitative mapping of the orientation of Fibroin $\beta$-sheets in B. mori cocoon fibers by scanning transmission X-ray microscopy, Biomacromol. 7, 836-843 (2006).

[4] K. Asakawa, S. Masuda, H. Takeuchi, Indole ring orientations of Trp189 in the ground and $M$ intermediate states of bacteriohodospin as studied by polarized UV resonance Raman spectroscopy, J. Raman Spectrosc. 37, 255-262 (2006).

[5] E. N. C. Mills, M. L. Parker, N. Wellner, G. Toole, K. Feeney, P. R. Shewry, Chemical imaging the distribution of ions and molecules in developing and mature wheat grain, J. Cereal Sci. 41, 193-201 (2005).

[6] D. M. Schulz, C. Ihling, G. M. Clore, A. Sinz, Mapping the toporogy and determination of a low-resolution threedimensional structure of the calmodulin-melittin complex by chemical cross-linking and high-resolution FTICRMS: Direct demonstration of multiple binding modes, Biochem. 43, 4703-4715 (2004).

[7] M. Sadeghi, X. Wu, A. Vertes, Conformation changes, complexation, and phase transition in matrix-assisted laser desorption, J. Phys. Chem. B, 105, 2578-2587 (2001).

[8] J. C. Vickerman, D. Briggs, TOF-SIMS: Suraface Analysis by Mass Spectrometry, IM Publications and SurfaceSpectra Ltd., UK (2001).

[9] S. Aoyagi, M. Hayama, U. Hasegawa, K. Sakai, M. Tozu, T. Hoshi, M. Kudo, TOF-SIMS Imaging of Protein Adsorption on Dialysis Membrane by means of Information Entropy, e-J. Surf. Sci. Nanotech. 1, 67-71 (2003).

[10] C. E. Shannon, W. Weaver, The mathematical theory of information, University of Illinois Press, Urbana, IL (1947).

[11] S. Aoyagi, Y. Kawashima, M. Kudo, TOF-SIMS imaging technique with information entropy, Nucl. Instr. Methods Phys. Res. B 232, 146-152 (2005).

[12] K. Eckschlager, V. Stepanek, K. Danzer, A review of information theory in analytical chemometrics, J Chemometrics 4, 195-216 (1990).

[13] H. U. Jabs, G. Assmann, D. Greifendorf, A. Benninghoven, High performance liquid chromatography and time-of-flight secondary ion mass spectrometry: A new dimension in structural analysis of apolipoproteins, J. Lipid Res. 27, 613-621 (1986).

[14] D. S. Mantus, B. D. Ratner, B. A. Carlson, J. F. Moulder, Static secondary ion mass spectrometry of adsorbed proteins, Anal. Chem. 65(10), 1431-1438 (1993).

[15] J. Davies, C. S. Nunnerley, A. J. Paul, A correlative study of the measurement of protein adsorption to steel, glass, 
polypropylene, and silicone surfaces using ToF-SIMS and dynamic contact angle analyses, Colloid. Surf. B 6, 181190 (1996).

[16] C. Poleunis, C. Rubio, C. Compére, P. Bertrand, Role of salts on the BSA adsorption on stainless steel in aqueous solutions. II. ToF-SIMS spectral and chemical mapping study, Surf. Interf. Anal. 34, 55-58 (2002).

[17] K. Leufgen, M. Mutter, H. Vogel, W. Szymczak, Orientation modulation of a synthetic polypeptide in selfassembled monolayers: A TOF-SIMS study, J. Am. chem. Soc. 125, 8911-8915 (2003).

[18] A. M. Belu, D. J. Graham, D. G Castner, Time-of-flight secondary ion mass spectrometry: Techniques and applications for the characterization of biomaterial surfaces, Biomaterials 24, 3635-3653 (2003).

[19] J. Xu, O. Ostrowski, C. Szakal, A. G Ewing, N. Winograd, ToF-SIMS imaging with cluster ion beams, Appl. Surf. Sci. 231-232, 159-163 (2004).

[20] M. S. Wagner, D. G. Castner, Characterization of adsorbed protein films by time-of-flight secondary ion mass spectrometry with principal component analysis, Langmuir 17, 4649-4660 (2001).

[21] N. Xia, D. G. Castner, Preserving the structure of adsorbed protein films for time-of-flight secondary ion mass spectrometry analysis, J. Biomed. Mater. Res. 67A, 179190 (2003).

[22] H. Wang, D. G. Castner, B. D. Ratner, S. Jiang, Probing the orientation of surface-immobilized immunoglobulin $G$ by time-of-flight secondary ion mass spectrometry, Lagmuir 20, 1877-1887 (2004).

[23] H. J. Mathieu, Bioengineered material surfaces for medical applications, Surf. Interf. Anal. 32, 3-9 (2001).

[24] S. Aoyagi, M. Hayama, U. Hasegawa, K. Sakai, M. Tozu, T. Hoshi, M. Kudo, Estimation of protein adsorption on dialysis membrane by means of TOF-SIMS imaging, J. Membr. Sci. 236(1-2), 91-99 (2004).

[25] S. Aoyagi and M. Kudo, Mutual Information Theory for
Biomedical Applications, Appl. Surf. Sci., (in press).

[26] S. Aoyagi, S. Hiromoto, T. Hanawa, M. Kudo, TOF-SIMS investigation of metallic material surface after culturing cells, Appl. Surf. Sci. 231-232, 470-474 (2004).

[27] H. E. Canavan, X. H. Cheng, D. J. Graham, B. D. Ratner, D. G. Castner, Surface characterization of the extracellular matrix remaining after cell detachment from a thermoresponsive polymer, Langmuir 21(5), 1949-1955 (2005).

[28] T. Waratrujiwong, B. Krebs, F. Spener, P. Visoottiviseth, Recombinant purple acid phosphatase isoform 3 from sweet potato is an enzyme with a diiron metal center, FEBS J. 273(8), 1649-1659 (2006).

[29] K. Saito, T. Kato, H. Takamori, T. Kishimoto, K. Fukushima, A new analysis of the depolymerized fragments of lignin polymer using ToF-SIMS, Biomacromol. 6(5), 2688-2696 (2005)

[30] M. C. Perkins, C. J. Roberts, D. Briggs, M. C. Davies, A. Friedmann, C. A. Hart, G.A. Bell, Surface morphology and chemistry of Prunus laurocerasus L. leaves: a study using $X$-ray photoelectron spectroscopy, timeof-flight secondary-ion mass spectrometry, atomic-force microscopy and scanning-electron microscopy, Planta 221(1), 123-134 (2005).

[31] S. Aoyagi and M. Kudo, Effective monitoring of protein reaction on glass plate surfaces by TOF-SIMS, Biosens. Bioelectron. 20(8), 1626-1630 (2005).

[32] B. X. Huang, H. Y. Kim, C. Dass, Probing threedimensional structure of bovine serum albumin by chemical cross-linking and mass spectrometry, J. Am. Soc. Mass Spectrom. 15(8), 1237-1247 (2004).

[33] S. Curry, H. Mandelkow, P. Brick, N. Franks, Crystal structure of human serum albumin complexed with fatty acid reveals an asymmetric distribution of binding sites, Nat. Struct. Biol. 5, 827-835 (1998). 